\title{
Los proyectos legislativos previsionales en Argentina y Chile durante el período 2005-2010. Un estudio comparado
}

Legislative prospects for social secutity reforms in Argentina and Chile. A comparative approach, 2005-2010

Francisco Oesterheld Licenciado en Sociología; Maestrando en Estudios Sociales Latinoamericanos, UBA foesterheld@live.com.ar
Fecha de recepción:

18.10.17

Fecha de aceptación:

23.2.18

\section{Resumen}

Los gobiernos de Néstor Kirchner (2003-2007) y Cristina Fernández (2007-2015) en Argentina y de Michelle Bachelet (2006-2010) en Chile heredaron sistemas previsionales en crisis; con problemas de financiamiento, cobertura y nivel de haberes. Ante la falta de cobertura se lanzaron programas para facilitar el acceso a la jubilación. Este tipo de medidas generaron debates en torno al funcionamiento general del sistema. El presente artículo versa sobre las estrategias y proyectos legislativos en materia previsional propuestos en Argentina y Chile durante el período 2005-2010. A partir de la perspectiva de la sociología histórica, compara las propuestas para mejorar la cobertura y el nivel de los haberes.

Palabras clave: Proyectos legislativos previsionales - cobertura de adultos mayores - haberes -

Abstract:

Both Néstor Kirchner's (2003-2007) and Cristina Fernández's (2007-2015) governemnts in Argentina and Michelle Bachelet's (2006-2010) in Chile inher- 
ited social security systems in crisis; with problems of $\mathrm{fi}$ nancing, coverage, level of retirement and, consequently, with poverty problems in older adults. In the absence to coverage shortcomings programs were launched to facilitate access to retirement. This type of measures generated an intense debate with regard to the overall working of the system. Appealing to the perspective of historical sociology, this article focuses on social security strategies and draft legislation in pensions advanced in both countries in 2005-2010. It compares proposals to improve coverage, level of retirement and, consequently, the distribution of income and the reduction of poverty.

Key words: social security legislative projects - distribution of income - poverty

\section{Introducción}

Las crisis previsionales heredadas por los gobiernos de Néstor Kirchner (2003-2007) y Cristina Fernández (2007-2015) en Argentina y Michelle Bachelet (2006-2010) en Chile, provinieron de la incapacidad de las reformas neoliberales implementadas en los años 1994 y 1981 -respectivamente- de aumentar las coberturas y los haberes. No obstante, los viejos sistemas públicos de reparto de ambos países, vigentes en Argentina a partir del año 1954 y en Chile desde 1952, tampoco habían podido generar elevados haberes en los sectores populares, aunque sí una amplia cobertura.

En Chile el haber promedio de los trabajadores manuales era, hacia $1980, \$ 5.024$ (a pesos de 1983), equivalentes a $\$ 128,82$ dólares, mientras la pensión mínima de vejez era de \$4.940 (126,67 dólares) y la pensión promedio general era de \$8.309 (213,05 dólares). La pensión promedio de los empleados particulares del sector privado era de $\$ 6.397(164,02$ dólares). Por otra parte, las pensiones asistenciales oscilaban entre un tercio y la mitad de la pensión mínima, dependiendo de los aportes del beneficiario (Arellano, 1985).

En Argentina el haber medio del Régimen Previsional Público había alcanzado un máximo de \$1.200 en 1934 (a precios constantes de 1994), es decir, \$1.200 dólares. Sin embargo, este elevado nivel del haber promedio se dio en el contexto donde menos del 7\% de la población económicamente activa estaba cubierta. Durante el peronismo (1943-1955), paralelo a la expansión de la cobertura, el haber medio tuvo un repunte 
alcanzando los $\$ 1.100$ dólares en el año 1947. De todas formas, a fines de la década de 1980 el haber medio apenas superaba los 200 dólares (Arceo et al., 2008).

Este deterioro de los ingresos, sumado a los elevados gastos previsionales en relación a los productos brutos internos respectivos, los déficits primarios, la crisis internacional del keynesianismo, sirvieron de fundamentación para justificar la privatización completa del sistema chileno y la privatización parcial del sistema argentino.

Esas reformas proponían resolver los problemas fiscales generados por los déficits previsionales de las respectivas cajas jubilatorias. Las transferencias presupuestarias de los estados eran cada vez mayores, a raíz de los procesos de maduración ocupacional, es decir, había cada vez más jubilados en relación a los trabajadores, lo que se agravaba a su vez por los procesos de envejecimiento poblacional a partir de la disminución de la tasa de natalidad y el aumento de la expectativa de vida.

Sin embargo, los sistemas privados también tuvieron dificultades para generar haberes dignos. Hacia marzo de 2008, previo a la reforma impulsada por el gobierno de $\mathrm{Mi}$ chelle Bachelet, el 14\% de los adultos mayores (256.000 personas) percibía las pensiones asistenciales (PASIS) de $\$ 48.000$ (90 dólares) para los adultos mayores de 65 a 70 años de edad; la pensión mínima garantizada por el estado (PMGE) era de \$96.390 (182 dólares) para los menores de 70 años (Superintendencia de Pensiones, 2010:12). El valor mediano de las pensiones autofinanciadas (otorgadas por las AFP) fue de $\$ 37.667$ en el período 2007-2014 (\$18.233 en el caso de las mujeres y $\$ 80.933$ en el caso de los hombres). ${ }^{1}$

A partir del 1 de julio de 2008, las PASIS fueron reemplazadas por las Prestaciones Básicas Solidarias (PBS), nuevo beneficio establecido por la reforma del 2008, con un monto inicial de $\$ 60.000$ (US\$113) para el $40 \%$ más pobre de la población; que aumentó a $\$ 75.000$ (US\$141) el 1 de julio de 2009, pasando a cubrir al 45 por ciento más pobre de la población; y con otros incrementos sucesivos hasta cubrir al 60\% más pobre de la población en el año 2011. La cobertura de los adultos mayores pasó, entre los años 2006 a 2008 , de un $79 \%$ a un $84 \%$; y, en el caso particular de las mujeres, pasó de un $74 \%$ a un 84\% en dicho período (Comisión Presidencial Pensiones, 2015: 85 y 87a).

En Argentina, durante la post convertibilidad, el haber mínimo logró recuperar el poder adquisitivo perdido tras la devaluación de 2002 e, incluso, superar el haber mínimo vigente durante la década de 1990. No obstante, no sucedió lo mismo con el haber medio. Si bien recuperó poder adquisitivo tras la devaluación de 2002, no superó el poder adquisitivo vigente durante la convertibilidad (Arceo et al., 2008:37-38). La cobertura de adultos mayores pasó, entre los años 2003 y 2010, de un 49,2\% (Cardozo, 2016: 103a) a un 95,8\% (ANSES, 2013: 59). En síntesis, las reformas a estudiar tuvieron la virtud de aumentar las coberturas de los adultos mayores, sin embargo, no tuvieron el mismo éxito en lograr mayores aumentos de los haberes. 


\section{La reforma previsional de Chile (2005-2010) El diagnóstico del Consejo Asesor Presidencial}

Tras ganar las elecciones presidenciales en 2006, Michelle Bachelet creó un Consejo Asesor Presidencial para la Reforma Previsional integrado por un grupo de 15 expertos designados por la Presidenta. En su mayoría, eran economistas muy prestigiosos del mundo académico, en algunos casos profesores universitarios, con títulos de posgrado en las Universidades más importantes del mundo, con cargos públicos importantes en los gobiernos anteriores. Director del Consejo fue designado Mario Marcel, un economista socialista muy cercano a Michelle Bachelet, que había sido subdirector de Presupuestos en el gobierno de Patricio Aylwin (1990-1994), entre otros cargos en las siguientes administraciones.

En primer lugar, la comisión tuvo como objetivo establecer un diagnóstico de la situación previsional, en el marco de las consecuencias de la reforma realizada durante la dictadura militar en 1981. Para ello realizó estudios y consultas a organizaciones sociales, organizaciones empresariales, institutos de estudio y expertos. Hubo intensos debates entre liberales y progresistas, principalmente en las audiencias públicas, sobre el fracaso (o no) de las Administradoras de Fondos y Pensiones (AFP). De acuerdo a algunos autores este consejo, dadas las características personales de sus miembros, garantizaba la permanencia del sistema privado (Garretón, 2012: 152).

El informe final del Consejo Asesor consideraba que la crisis de cobertura no se debía al fracaso de la reforma del régimen del general Pinochet. Argumentaba la falta de un proceso de maduración de las AFP y, por lo tanto, no buscaba realizar una reforma estructural del sistema. Es decir, no había una crisis endógena del sistema previsional: las cotizaciones de los trabajadores en relación de dependencia se realizaban de manera regular, las AFPs cumplían con sus obligaciones legales, las rentabilidades de los fondos de inversión habían sido siempre positivas durante los 25 años de existencia del sistema, a excepción de dos años, y ninguna AFP había quebrado ni cometido fraudes. La mayoría de los jubilados habían sido afiliados del sistema antiguo o beneficiarios de las pensiones asistenciales. La mayoría de los afiliados provenía del sistema antiguo y, en consecuencia, percibía el bono de reconocimiento. Por lo tanto, hacía falta el transcurso de otros 20 años más para la plena madurez del sistema con una mayoría de jubilados genuinos del sistema privado de capitalización individual. A su vez, muchos trabajadores y trabajadoras no confiaban en el sistema jubilatorio como garante de una vejez sin pobreza. Por lo tanto, no ahorraban lo suficiente para cumplir con la normativa dispuesta en el decreto ley 3.500 .

El informe no negaba la crisis de cobertura previsional, sobre todo en la población económicamente activa (PEA), evidenciada por la realidad misma y diferentes estudios 
al respecto. Asimismo, constataba los fuertes cambios acaecidos en los 25 años transcurridos desde la reforma de 1981 en materia de demografia, mercado laboral y en la estructura social. En Chile, lo mismo que el resto de la región, se registraba un proceso de envejecimiento demográfico, donde la población mayor de 60 años pasó de representar 7,5\% de la población total en el año 1960, a un 11,5\% en el 2005, acelerándose el proceso de envejecimiento según algunas proyecciones para los años 2020 y 2050, con una población mayor del $17,3 \%$ y $28,2 \%$ respectivamente. A su vez, la tasa de natalidad comenzó a descender abruptamente a partir de la década de 1960. Esto complejizaba todavía más la situación de los adultos mayores, al formar parte de familias con menor cantidad de hijos, e incluso de estructuras monoparentales en muchos casos, lo que se traducía en una menor red de apoyo, tanto en términos de cuidados como de transferencias de ingresos intrafamiliares.

Con respecto al mercado de trabajo, en las últimas décadas se había suscitado un aumento sustantivo de la informalidad laboral; agravada todavía más por una mayor participación de las mujeres, quienes padecen situaciones de mayor inestabilidad. Por lo tanto, al deteriorarse los contratos fijos por tiempo indeterminado predominantes en otras épocas, se veía erosionada la capacidad de cotización de los afiliados (Resumen Ejecutivo Informe Consejo Reforma Previsional, 2006: 4 y 5a).

\section{Las propuestas de la nueva reforma}

El objetivo final de la nueva reforma consistía en la creación de un sistema solidario no contributivo, administrado y financiado por el estado, complementario del sistema privado, sin ningún tipo de perjuicio a éste. En tal sentido, la decisión política de la Presidenta de la Nación era conformar un gran sistema de protección social, trascendiendo los conflictos entre liberales y progresistas. Esto era, por un lado, no modificar el sistema privado de capitalización individual, satisfaciendo a sus defensores, entre los que estaban los funcionarios de ideología liberal y los grupos más concentrados de las finanzas, principales beneficiarios del sistema previsional neoliberal. Por otro lado, crear un sistema solidario administrado por el estado, permitiendo el acceso a la jubilación a todas aquellas personas que no cumpliesen con los 20 años de aportes mínimos exigidos por el decreto ley 3.500 de 1980 . De esta manera, ampliar la cobertura previsional para los adultos mayores, minimizando los riesgos de caer en la pobreza.

El sistema previsional debía estar integrado por tres pilares: el contributivo, el voluntario y el nuevo sistema solidario. Estaría regido, en primer lugar, por el principio de universalidad, garantizando a todos los mayores una cobertura, independientemente de su trayectoria laboral. En segundo lugar, debía respetarse el principio de la equidad social, favoreciendo a aquellos trabajadores con más aportes al sistema contributivo, pero respetando el principio de autonomía, es decir, garantizando pensiones solidarias de un monto 
suficiente para minimizar los riesgos de la pobreza en la vejez. Por último, todos los actores eran responsables del buen funcionamiento del sistema, tanto las administradoras privadas como los afiliados. Sin embargo el responsable en última instancia era el estado, quien debía contar con las facultades de fiscalización, regulación y recursos para compensar las falencias del sistema, garantizando así los derechos establecidos en la Constitución.

Una vez realizado el diagnóstico surgieron 70 propuestas de reforma distribuidas en las siguientes problemáticas: a) nuevo pilar solidario; b) cobertura, densidad de cotizaciones y cumplimiento del mandato de cotizar; c) equidad de género; d) competencia y organización de la industria de AFP; e) competencia y precios; f) inversión de los fondos de pensiones; g) pensiones en el pilar contributivo; h) ampliación del pilar voluntario; i) educación e información;j) institucionalidad; k) disciplina financiera.

El nuevo pilar solidario se estructuraría en torno a un nuevo beneficio: la pensión básica solidaria (PBS). Este ingreso sería asignado a aquellas personas que no obtuviesen ninguna pensión autofinanciada del sistema contributivo; y a quienes tuviesen algunos fondos acumulados, pero asignándose de manera decreciente a mayor monto de la pensión financiada, quedando completamente absorbido ante una pensión autofinanciada de $\$ 200.000$. Asimismo, los beneficiarios debían pertenecer al $60 \%$ más pobre de la población. Esta medida benefició principalmente a los trabajadores independientes, a las trabajadoras mujeres con menor estabilidad laboral que los hombres, y los demás trabajadores de bajos ingresos. De todas formas, respetaba el principio de equidad social mencionado más arriba, al premiar a los afiliados con ahorros previsionales, permitiéndoles sumar esos ahorros a la pensión final.

La pensión básica solidaria (PBS) reemplazó a las pensiones asistenciales (PASIS) y a la pensión mínima garantizada por el estado (PMGE). Las PASIS eran de un monto ínfimo, nunca habían estado integradas al sistema general y para acceder a ellas era necesaria una compleja evaluación socio-económica de las familias. Por otro lado, la pensión mínima garantizada por el estado, creada en el decreto ley 3.500 de 1980, garantizaba la pensión mínima para aquellos afiliados que hubiesen aportado un mínimo de 24 meses, y no alcanzaran a obtener la pensión mínima del sistema privado.

En julio del 2006, el informe llegó a manos de Michelle Bachelet, quien creó un comité interministerial, conformado por los ministros de Trabajo y de Hacienda, Osvaldo Andrade y Andrés Velasco, y por la Secretaria General de la Presidencia y la titular del Servicio Nacional de la Mujer (SERNAM), Paulina Veloso y Laura Albornoz respectivamente. Este comité estuvo a cargo de la definición del cuerpo legal del proyecto, finalmente enviado al Congreso a fines del 2006. 


\section{Ley 20.255}

El 11 de marzo de 2008 se promulgó la ley 20.255, mediante un decreto firmado por Michelle Bachelet. Quedó establecido en el artículo 1 la creación de un sistema solidario, complementario al sistema vigente, otorgando el beneficio de la pensión básica solidaria de vejez e invalidez (PBS) y el aporte previsional solidario (APS) de vejez e invalidez.

En consonancia a las propuestas del Consejo Asesor, la PBS quedó definida como el beneficio otorgado hacia aquellas personas que cumplieran con los requisitos establecidos en el artículo $3^{\circ}$ : no tener derecho a ninguna pensión del sistema vigente, ser mayores de 65 años, pertenecer a una familia calificada dentro del 60\% más pobre de la población, contar con una residencia en el país no menor a los 20 años ya sea en forma consecutiva o interrumpida -computándose a partir de sus 20 años de edad-, y de los últimos 5 años haber residido por lo menos 4 .

Por otra parte, se define el concepto de pensión base (PB) como el resultado de la sumatoria de la pensión autofinanciada y la pensión de sobrevivencia en caso de existir, ambas establecidas por el decreto ley 3.500 del año 1980. Por último se define el concepto de aporte previsional solidario.

En resumen, el APS es un beneficio asignado por aquellas personas que cumplan con los requisitos establecidos en el párrafo tercero del título I de la ley. Al contrario de la PBS, el APS sería percibido por aquellas personas con derecho a una o más pensiones del sistema previsional vigente, es decir, tener 20 años mínimos de aporte y obtener una pensión inferior a la mínima; siempre y cuando cumplan con los requisitos establecidos para la PBS, es decir, pertenecer a una familia ubicada dentro del $60 \%$ de la población más pobre, ser mayores de 65 años y computar 20 años de residencia en el país.

Dicho esto, una vez definidos los beneficios más importantes de la reforma, la PBS y el APS, es necesario entrar en algunos detalles, de otros conceptos importantes definidos en el artículo $2^{\circ}$, que hacen al cálculo de las pensiones con derecho al APS. Por una parte, la pensión máxima con aporte solidario (PMAS), es el monto sobre el cual la pensión base no tiene aporte previsional solidario. En este sentido, el artículo $11^{\circ}$ realiza algunas aclaraciones: dentro de los beneficiarios del APS establecidos en el artículo $9^{\circ}$, aquellos que tengan una pensión base superior a la pensión básica solidaria (PBS) e inferior al PMAS, el monto del APS variará según las siguientes situaciones: en caso de percibir una pensión en la modalidad de renta vitalicia, conforme a lo establecido por el decreto ley 3500, el monto del APS será equivalente al complemento solidario. Si, por el contrario, la persona recibe una pensión en la modalidad de retiro programado, el monto del APS será equivalente al monto del complemento solidario pero corregido por un coeficiente de ajuste. 
Por otra parte, quedan por definir los conceptos de factor de ajuste y complemento solidario. El primero es resultado de dividir la pensión básica solidaria por la pensión máxima con aporte previsional solidario. El segundo, utilizado para calcular el monto del APS, es igual a la pensión básica solidaria menos el producto obtenido de la multiplicación entre el factor de ajuste y la pensión base.

La Comisión Asesora Presidencial estimaba que, de seguir el sistema de las AFP sin la reforma propuesta, las tasas de sustitución del salario serían del 44\%; y como consecuencia de la implementación de la reforma alcanzarían el 70\%. Dentro de los sectores de menores ingresos, la tasa de sustitución del salario (TSS) fue la más incrementada, permitiendo una menor desigualdad entre los jubilados. Para el 20\% con menor acumulación de ahorro, sus TSS alcanzan el 80\%, superando el promedio general. En el caso de las mujeres, sus TSS se duplican, alcanzando el mismo nivel de los hombres. Asimismo, la reforma permitiría ampliar la cobertura, garantizando una pensión mínima superior al ingreso per cápita de pobreza. Si bien no le garantizaba al adulto mayor dejar de pertenecer a un hogar pobre, le permitía a él solo con su propio ingreso salir de la pobreza individual. Estos mayores beneficios se financiarían duplicando el gasto fiscal representado por las pensiones asistencias y las pensiones mínimas garantizadas por el Estado, alcanzando un 1\% del PBI del año 2025. Este incremento del gasto fiscal sería compensado por el gasto decreciente demandado por el sistema antiguo de pensiones (Resumen Ejecutivo Informe Consejo Reforma Previsional, 2006: 29b).

\section{Los resultados de la reforma}

Hasta el año 2016, en Chile había un total de 1.594.403 adultos mayores de los cuales 1.178.185 recibían algún tipo de beneficio previsional (73,9\% de la cobertura) (Cardozo, 2016: 104b). Para alcanzar una noción de las proporciones en que se distribuyen las distintas prestaciones, presentamos a continuación algunos datos de la Superintendencia de Pensiones publicados recientemente en el mes de diciembre de 2017. Allí se constata, en primer lugar, que el sistema público solidario no contributivo pagaba íntegramente 581.908 pensiones tanto de vejez como invalidez. Es decir, el Estado pagaba al 100\% estas pensiones asignadas a personas que no tenían derecho a la pensión privada por no disponer de los 20 años de requisito mínimo contributivo establecido por el decreto-ley 3.500 del año 1980.

En segundo lugar, 872.587 personas cobraban pensiones autofinanciadas (pagadas por las AFP) y a su vez cobraban el aporte solidario previsional (pagado por el sistema público no contributivo).Las AFPs pagaban íntegramente las jubilaciones sólo del 4,3\% de los adultos mayores. En cambio, el Estado, teniendo en cuenta el pago de las pensiones del derogado sistema de reparto y del sistema solidario no contributivo, pagaba -de manera total o parciallas jubilaciones de las tres cuartas partes de la población adulta mayor (Cardozo, 2016: 104c). 
La fundación SOL publicó un estudio donde constataba que la mayoría de los pensionados chilenos de las AFPs percibían una jubilación menor a $\$ 147.000$ (257,72 dólares). $87,3 \%$ de los hombres y el $93,1 \%$ de las mujeres cobraba menos de $\$ 147.000$, mientras el sueldo mínimo era de $\$ 225.000$ (biobiochile.cl, 2015). Por otro lado, la Prestación Básica Solidaria (PBS) en el año 2014, representaba tan sólo el 38\% del Salario Mínimo nacional, pero se ubicaba por encima de la línea de pobreza por persona, aunque sea de manera marginal en 1,06 veces (Fundación SOL, 2014: 6). La Comisión Bravo también constata que el $80 \%$ de las personas recibiría una pensión inferior al salario mínimo, y un $44 \%$ percibe ingresos por debajo de la línea de pobreza.

Con respecto a la cobertura previsional de los adultos mayores, la encuesta CASEN (2013) informó que el 84,5\% de los mayores de 65 años perciben pensiones contributivas o no contributivas. El 70\% fueron pensiones de vejez; el 12,5\% pensiones de sobrevivencia o viudez; y el 2,7\% pensiones de invalidez. La cobertura previsional, luego de la reforma, pasó de un 79\% en el año 2006 a un 84\% en el año 2008. En las mujeres, el aumento fue mayor, pasando de un 74\% a un 84\%, entre los años 2006 y 2013. Este aumento fue generado, fundamentalmente, por la creación de la Prestación Básica Solidaria (PBS), al cubrir para el año 2015 al 30\% de la población mayor de 65 años; donde la cobertura femenina de PBS es de $36,3 \%$, y la cobertura masculina de PBS es de 22,3\% (Informe Bravo, 2015: 81).

En lo que respecta a la situación de pobreza en los adultos mayores, para el año 2013, según la metodología tradicional, el 3,9\% de los mayores de 60 años era pobre, y según la metodología nueva el 8,5\% eran pobres. En cuanto a la presencia de adultos mayores en los hogares, lo cual está estrechamente vinculado a la situación de pobreza (o no), el 36\% de los hogares vive con presencia de al menos una persona mayor de 60 años. El 50\% de las personas mayores vive en un hogar donde el jefe de hogar también es una persona mayor. El 13\% de las personas mayores de 60 años vive en hogares unipersonales, 34\% en hogares bipersonales y $22 \%$ en hogares tripersonales. En los hogares con personas mayores hay un menor índice de pobreza en comparación de los hogares donde no hay adultos mayores. En este sentido, el 17\% de los hogares sin personas mayores es pobre; el $17 \%$ de los hogares con personas mayores y personas menores de edad son pobres; y el 10\% de los hogares conformados solamente por personas mayores son pobres (Comisión Presidencial Pensiones, 2015: 61b).

La nueva metodología de ingresos para medir la pobreza, implementada a partir del año 2015 , establece una línea de pobreza de $\$ 368.389$ (en valores de abril 2012) para un hogar (promedio de 4,43 integrantes), equivalente a una línea de pobreza por persona de \$129.964 La pensión básica solidaria a partir de julio era de $\$ 85.964$ mensuales, es decir, por debajo de la línea de pobreza por persona. 


\section{La reforma previsional de Argentina (2005-2010)}

El 25 de mayo del 2003 Néstor Kirchner asumió como Presidente de la Nación. La Argentina estaba saliendo de una de sus peores crisis económica, política y social. La deuda externa representaba el 160\% del Producto Bruto Interno, la pobreza había alcanzado un 54\% de la población luego de la devaluación del año 2002 durante la Presidencia de Eduardo Duhalde. La declaración de default de Adolfo Rodríguez Saá significó el inicio de una nueva política ante la deuda. En este sentido, antes de asumir formalmente la presidencia el ex gobernador de Santa Cruz, hubo un debate en el seno de los cuadros técnicos del Frente Para la Victoria: en torno a implementar una política de desendeudamiento, o comenzar la gestión con un nuevo préstamo de 20 mil millones de dólares ante el bajo nivel de reservas del Banco Central (Moreno, 2017: 27). El Presidente decidió la primera opción y, por otro lado, estableció tres pilares fundamentales del posterior crecimiento económico: el tipo de cambio competitivo, la baja de las tasas de interés y el incremento de los ingresos populares. ${ }^{2}$

Ante un proceso de reactivación de la economía, en un contexto internacional favorable para las commodities del país, se comenzaron a gestar los primeros debates en torno a la restitución de los derechos previsionales, sumamente vulnerados en las décadas anteriores. El eje de la discusión giró en torno a dos caminos a elegir para mejorar la situación previsional: universalizar la cobertura de los adultos mayores o recomponer los haberes medios luego de décadas de pérdidas del poder adquisitivo, ante los procesos hiperinflacionarios y por último ante la devaluación que había conducido al fin del régimen de la convertibilidad.

El gobierno nacional tomó como prioridad universalizar la cobertura, constituyendo un sistema con una cobertura que llegó a alcanzar el 97\% en 2015, pero con un 53\% de los pensionados percibiendo haberes mínimos.

\section{La jubilación anticipada y las moratorias previsionales}

En términos legislativos, este sendero se fue forjando mediante la Ley 25.994, promulgada en el año 2004. Estableció una jubilación anticipada para varones mayores de 60 años y mujeres mayores de 55, con 30 años de aportes computables, y que acreditaran estar en desempleo al 30 de noviembre del 2004. El monto de la prestación representaría el 50\% de su pensión correspondiente, no pudiendo ser inferior al haber mínimo del sistema público de reparto. Luego, una vez cumplida la edad mínima exigida por la ley 24.241, podían pasar a cobrar el $100 \%$.

La nueva normativa tenía un carácter transitorio; vencía a los dos años, con la posibilidad de prorrogarse. De todas formas, mediante la Resolución 1451/2006, se renovó su vigencia hasta el 30 de abril del año 2007, y mediante la Resolución 1054/2006 se extendió el requisito de estar desempleado al 31 de diciembre del 2005. 
A su vez, estableció en el artículo 6, la primera moratoria lanzada por el kirchnerismo, conforme a la Ley 25.865. En el año 2006, se amplió esta moratoria permitiendo regularizar las deudas previsionales vigentes hasta septiembre de 1993, a pagarse en 60 cuotas con una baja tasa de interés tanto para los trabajadores en relación de dependencia como para los monotributistas y cuentapropistas. Esto significaba que, por ejemplo, en el caso de una persona en edad de jubilarse para el año 2006, sin aportes entre los años 1990 y 2000 , los primeros tres años eran reconocidos con la moratoria con una fuerte quita y la posibilidad de pagarse en 60 cuotas, mientras el resto de los años adeudados debían pagarse íntegramente y en efectivo (Página 12,5/6/2014). Estas medidas permitieron el acceso a la jubilación de 2,6 millones de adultos mayores. En el año 2014, se sancionó la segunda moratoria, durante la presidencia de Cristina Fernández de Kirchner, permitiendo el acceso a la jubilación a 500.000 personas más, estableciendo el año 2003 como fecha límite para regularizar las deudas previsionales.

Los beneficiarios de la jubilación anticipada fueron 44.161 personas, que obtuvieron el beneficio entre los años 2006 y 2008, hasta vencer la normativa (Madera, 2011: 11). En cambio, los beneficiarios de las moratorias previsionales fueron sustancialmente mayores, superando el millón de nuevos beneficiarios entre los años 2006 y 2007. Había dos tipos de moratorias previsionales: la moratoria previsional permanente (MPP); y la moratoria previsional excepcional (MPE) con vigencia sólo hasta 2007, y con menor exigencia para obtener la jubilación. Ésta última fue la que explicó el incremento repentino de la cobertura.Ambas moratorias se establecieron por la resolución conjunta 579/2006 del ANSES, y la resolución conjunta 2091/2006 de la AFIP (Madera, 2011: 11 y 28).

\section{Las propuestas del arco opositor}

En un contexto de resultados insatisfactorios por parte de las Administradoras de Fondos de Jubilaciones y Pensiones (AFJPs), desde diferentes sectores de la oposición política no se habían cumplido, comenzaron a impulsarse varias propuestas de reforma, entre los años 2003 y 2008. Los proyectos giraban en torno a aumentar las jubilaciones y pensiones, desde actualizaciones de los beneficios mediante la constitución de índices de costo de vida, propuesto por los propios defensores de las AFJP. Los técnicos expertos en el tema previsional, de las distintas fuerzas políticas, explicaron la imposibilidad de actualizar las jubilaciones por el costo de vida. Se presentaron 12 proyectos para plantear una movilidad de las jubilaciones en relación al aumento de los salarios, como había sido en el viejo sistema público de reparto y quebrado desde fines de los años setentas por el proceso de achatamiento continuo de la pirámide de ingresos.

Aunque finalmente se alcanzó un régimen de movilidad jubilatoria, se desarrolló un amplio debate en torno a la formulación del índice para calcular y aplicar los aumentos. El oficialismo propuso una fórmula con dos tramos, uno atada a los aumentos salariales 
y otro a los aumentos del presupuesto de la seguridad social. Según las visiones críticas de un informante clave, asesor previsional de un sector de la oposición, se determinaba el aumento a favor del índice que menos diera. Los aumentos de las jubilaciones finalmente estuvieron atados al aumento de los salarios, es decir, a las paritarias y por lo tanto al alza de los salarios de la población activa empleada.

\section{El Fondo de Garantía Sustentable}

En el año 2007 el decreto 897 creó el Fondo de Garantía. El FGS se formó con el objetivo de tener ahorros previsionales para poder seguir pagando las prestaciones en un año recesivo de la economía. El artículo 6 del decreto estableció un "tope", es decir, un techo a la cantidad de recursos que podía tener este fondo, que no podía superar un monto total equivalente al pago de un año entero de jubilaciones. No obstante, al año siguiente, con la re-estatización de las AFJPs mediante la Ley 26.425, ese artículo fue derogado quitándose el tope del FGS.

Según los sectores opositores, esta derogación del tope impedía aumentos más ambiciosos de las jubilaciones, porque permitía al Ejecutivo utilizar los excedentes obtenidos de la re-estatización de las AFJPs para otros fines como por ejemplo la Asignación Universal por Hijo o el pago de las moratorias. En cambio de permanecer el tope, hubiese obligado al Ejecutivo a destinar los excedentes para aumentar en mayor medida los haberes medios.

En este sentido, la mayoría de la oposición se opuso a "destopear" los fondos del FGS, dado que aspiraba a que los ingresos provenientes de las AFJPs fueran volcados en mayores aumentos de los haberes, y no a realizar una capitalización dentro del Estado más allá de un fondo suficiente para el pago de las jubilaciones durante un año. El posterior debate en torno al proyecto del $82 \%$ por ciento móvil respecto del sueldo básico de un trabajador activo, tanto el dictamen de minoría del sector del bloque legislativo conformado por Proyecto Sur, Libres del Sur y Nuevo Encuentro, como el dictamen de mayoría del denominado "Grupo A" de oposición, planteaban el financiamiento de $82 \%$ por ciento móvil por medio de los fondos recuperados de las AFJP, que podrían ser volcados en aumentos, siempre y cuando no se permitiese el "destope" del FGS. ${ }^{3}$

\section{La reestatización de las AFJPs y el 82\% móvil}

La ley 26.425 re-estatizó en 2008 AFJPS, derogando el Sistema Integrado de Jubilaciones y Pensiones instaurado en 1994 mediante la ley 24.491, y reemplazándolo por un sistema público de reparto denominado Sistema Integrado Previsional Argentino (SIPA).

Esta medida significó, entre otras cuestiones, que el dinero de los trabajadores y jubilados administrado por las AFJPs pasara a ser administrado por el estado por medio de 
la Administración Nacional de Seguridad Social (ANSES). Esto incrementó todavía más los fondos de las arcas del estado, que nunca había dejado de administrar el sistema previsional público, aunque había sido muy perjudicado al hacerse cargo del pago de las prestaciones básicas universales y prestaciones compensatorias, tanto de los afiliados del sistema público como del privado, y para colmo obteniendo la menor porción de la recaudación de la Seguridad Social.

Una porción del arco opositor constituida por sectores de centro-izquierda con representación parlamentaria apoyó la sanción de la ley, con algunas disidencias en torno a determinados artículos, sobretodo uno de los artículos finales que, como mencionamos más arriba, derogaba el "tope" del FGS.

En el año 2010, el arco opositor impulsó el proyecto del 82\% móvil de las jubilaciones con relación al salario básico. Sobre este punto es pertinente hacer un paréntesis. El debate sobre este tema es histórico. En el año 1958, durante la Presidencia de Arturo Frondizi, se estableció ese criterio para todas las jubilaciones, mediante la promulgación de la Ley 14.499. Esta medida generó el primer déficit del sistema previsional en 1962. Asimismo, derogó uno de los aspectos más progresivos del sistema previsional del primer peronismo que, mediante la Ley 14.370, establecía tasas de sustitución de salario del $100 \%$ para los sectores de menores ingresos. Es decir, en lugar de establecer el 82\% para los haberes mínimos, medios y altos, establecía el 100\% para los haberes mínimos, e iba decreciendo en términos porcentuales la tasa de sustitución de salario a medida que los salarios y las jubilaciones fueran más elevados en términos absolutos.

Durante las décadas de 1970 y 1980 los aumentos de las jubilaciones dejaron de estar atados íntegramente al aumento de los salarios. El primer achatamiento de la pirámide de jubilaciones se produjo durante el gobierno de Raúl Alfonsín (1983-1989), al otorgarse aumentos mínimos del 10\%. Por ejemplo, si los salarios aumentaban en un 40\%, se otorgaban aumentos del $10 \%$ a las jubilaciones más altas y del 25\% a las jubilaciones más bajas. Esta situación generó una sucesiva ola de juicios previsionales contra el estado. A raíz de la crisis financiera del sistema previsional, surgen los bocones, es decir bonos previsionales emitidos por el Estado que permitieron financiar el pago de las jubilaciones. De esta forma, el déficit previsional contribuyó al incremento de la deuda pública y al colapso institucional hacia fines de la década de los 80s. Por supuesto, la crisis fiscal no se explica sólo por el déficit previsional, sino fundamentalmente por la estatización de la deuda privada durante el golpe cívico-militar, el estancamiento de la economía a lo largo de todo el gobierno de Raúl Alfonsín y el exceso de emisión monetaria sin un crecimiento económico que lo respaldara.

En esta etapa de fuerte achatamiento de la pirámide de las jubilaciones se rompe con el viejo sistema jubilatorio, donde las jubilaciones estaban más atadas a los salarios, y 
comienza a constituirse un sistema que priorizaba a los sectores de menores ingresos, y perjudicaba a los sectores medios y altos de la pirámide de ingresos.

Este paréntesis histórico tiene muchísimo que ver con el debate durante la etapa del kirchnerismo. El dictamen del sector de la centro-izquierda del Congreso, proponía establecer el 82 por ciento móvil a los haberes mínimos y medios; estableciendo una propuesta de financiamiento con mayores cargas patronales. A su vez, sostenía que el 82 por ciento móvil propuesto por el dictamen del "Grupo A", no era viable y sustentable en términos financieros al proponer también el 82 por ciento para los haberes altos (Asesor Previsional de Proyecto Sur, comunicación personal, 2014a). Para el oficialismo, el 82 por ciento móvil de los haberes mínimos era el "mascarón de proa" del proyecto, fuertemente difundido por los medios masivos de comunicación. No obstante, el artículo 1 del proyecto de ley, que establecía el 82 por ciento móvil para los sectores de menores ingresos, era el que representaba menores erogaciones para el estado. La principal preocupación del oficialismo, eran los artículos 5, 6 y 7, al representar erogaciones mucho mayores en beneficio de los sectores medios y altos; incrementando aún más la desigualdad al interior del sistema. Por último, el oficialismo rechazaba el artículo 12 del proyecto opositor, al establecer un plazo de 30 días para que ANSES se despojara de los fondos sobrantes en relación a la partida asignada por el Presupuesto Nacional para la Seguridad Social. En este punto, aducía que la misma oposición, que ahora proponía que el estado desinvirtiera en un mes sus fondos, se había opuesto al artículo de la Ley de Medios que le daba un plazo de un año al Grupo Clarín para vender parte de su cartera. ${ }^{4}$ El argumento central del oficialismo para rechazar el proyecto de ley del 82 por ciento móvil, posteriormente vetado por el Poder Ejecutivo (Decreto 1482/2010) insistía en el efecto de desfinanciamiento de las arcas del estado que el proyecto opositor implicaba, no sólo una crisis al interior del sistema previsional público del conjunto del sector público por la vía del desfinanciamiento, y de la economía nacional al generar mayor inflación, devaluación, aumentos en la tasa de interés y déficit fiscal.

\section{Algunas conclusiones}

Las reformas previsionales recientes desarrolladas en Argentina y en Chile, han significado un avance importante en materia de aumento de la cobertura de los adultos mayores. Sin embargo, el nivel de las jubilaciones y pensiones de los sectores populares, es decir, los sectores mayoritarios, siguen siendo bajos. Por supuesto, desde un punto de vista, la diferencia entre no percibir una jubilación y percibir una, aunque sea el haber mínimo, significa una sustancial mejora para el beneficiario sobre todo si pertenece a los sectores sociales más vulnerables. Asimismo, significa una mejora en la distribución del ingreso en el conjunto de la sociedad, al otorgarse millones de jubilaciones nuevas no reconocidas por la normativa anterior. Al mismo tiempo, el hecho de que la mayoría de los jubilados cobren haberes mínimos o haberes medios todavía muy bajos alimenta algunas críticas. 
En el caso argentino, algunas opiniones consideran que no hubo una distribución del ingreso, sino del salario entre los propios trabajadores y/o jubilados; al argumentarse que la universalización de las jubilaciones se hizo en detrimento de una mayor recomposición de los haberes medios. Es decir, ante los recursos disponibles por el Estado, había dos opciones a elegir: universalizar la cobertura focalizándose en el mayor aumento de los haberes mínimos; o priorizar la recomposición de los haberes medios deteriorados en las últimas décadas y dejando en un segundo orden de prioridad la cuestión de la ampliación de la cobertura y el mayor aumento de los haberes mínimos (Asesor Previsional de Proyecto Sur, comunicación personal, 2014). En este sentido, había propuestas para obtener ambos objetivos, precisamente en el proyecto de ley del 82 por ciento móvil del dictamen de minoría, pero para ello se proponían reformas impositivas que podían atentar contra la inversión privada y el consecuente dinamismo de la economía.

En el caso chileno, algunas opiniones consideran pertinente establecer un sistema público de reparto que permitiría un mayor aumento de los haberes. En la actualidad el sistema público solidario no contributivo se encarga de los mayores gastos del sistema, mientras las AFPS que pagan una parte menor del gasto obtienen la "crema del negocio" al ser el sistema oficial obligatorio que monopoliza la obtención de los aportes y comisiones que pagan los afiliados que componen la población económicamente activa registrada (Cardozo, 2016: 125d).

Los sistemas previsionales privados han mostrado serias dificultades en su intento por mejorar los haberes y las coberturas. Los sistemas públicos de reparto o solidarios vuelven a demostrar su relevancia como garantía de inclusión para los sectores excluidos del mercado de trabajo formal y del sistema previsional contributivo. No obstante, también han mostrado cierto límite para otorgar pensiones de mayor cuantía para los sectores populares. Si bien su rol como garantes de un piso de dignidad es clave, también plantean posteriores reflexiones a la hora de intentar mejorar tanto los haberes mínimos como los haberes medios. 
${ }^{1}$ El monto de $\$ 37.667$ es la $<<$ pensión autofinanciada $>>$, es decir, la pensión otorgada por las AFP a las personas en función de la capitalización de sus cuentas individuales. En caso de que el beneficiario tuviese un mínimo de 240 meses de aportes, recibía los aportes de la pensión mínima garantizada por el estado vigente hasta el año 2008, o el Aporte Previsional Solidario vigente a partir del 2008. Sumando el APS a la pensión autofinanciada la mediana de las pensiones da $\$ 82.650$ en el período 2007-2014.

${ }^{2}$ Ver la participación de Guillermo Moreno, ex secretario de comercio interior durante los gobiernos de Néstor Kirchner y Cristina Fernández, en el programa televisivo 678 el 20 de diciembre 2015. Disponible en https://www.youtube.com/watch?v=g_2KtWGSQc0\&t=61s (en el minuto 45).

${ }^{3}$ El "Grupo A" estaba conformado por la UCR, el PRO, la Coalición Cívica de Elisa Carrió, el Peronismo Federal presidido por Felipe Solá, el GEN presidido por Margarita Stolbizer y el socialismo.

${ }^{4}$ Demian Panigo, 2010, entrevista recuperada de http://www.nuevaszonceras.com.ar/noticias/82-movilcomo-esta-ley-vacia-la-anses) 


\section{Referencias bibliográficas}

ANSES, Administración Nacional de Seguridad Social (2011). Marco conceptual del sistema de estadísticas e indicadores del sistema integrado previsional argentino: Observatorio de la Seguridad Social. Buenos Aires: 2da edición.. http://observatorio.anses.gob.ar/archivos/documentos/Cuadernillo_MARCO\%20CONCEPTUAL.pdf accesado el 17 de enero de 2017.

Arceo, N., González, M., Mendizábal, N. (2009). La evolución del sistema previsional argentino (Documento de Trabajo Número 2). Buenos Aires: CIFRA. http://www.aaps. org.ar/pdf/area_politicassociales/Basualdo.pdf accesado el 19 de febrero de 2017.

Arenas de Mesa, A. (2010). Historia de la reforma previsional chilena. Una experiencia exitosa de política pública en democracia. Suiza, Ginebra: OIT. http://www.ilo.org/wcmsp5/groups/public/---americas/---ro-lima/---sro-santiago/documents/publication/ wcms_178562.pdf accesado el 25 de mayo de 2017.

Cárcamo, H. (27 de junio de 2017). “Las distorsiones y vacíos que ocultan las estadísticas sobre jubilaciones reveladas por la Superintendencia de Pensiones”. El Mostrador. Recuperado de: http://www.elmostrador.cl/mercados/2017/01/06/las-distorsionesy-vacios-que-ocultan-las-estadisticas-sobre-jubilaciones-reveladas-por-la-superintendencia-de-pensiones/ accesado el 9 de abril de 2017.

Cardozo, N. (2016). “Los sistemas previsionales de la Argentina y Chile (2006-2010). Un estudio comparado”. Estado Abierto. Revista sobre el Estado, la administración y las políticas públicas, Vol. 1 Número 1, pp. 84-140. http://www.economicas.uba.ar/wp-content/ uploads/2016/07/ESTADO-ABIERTO-Vol-1-N-1.pdf accesado del 5 de marzo de 2017.

Comisión Asesora Presidencial sobre el Sistema de Pensiones (2015) Informe Final http://www.comision-pensiones.cl/Informe_final_CP_2015.pdf

Fiscella, S. (2003). “Ciudadanía y previsión social”. Gaceta Laboral, vol.9, núm. 3, septiembre-diciembre, pp. 353-395. http://www.redalyc.org/pdf/336/33609303.pdf accesado el 5 de mayo de 2017.

Fundación SOL (2014). Presentación de la Fundación SOL para Comisión Asesora Presidencial sobre el Sistema de Pensiones. http://www.nomasafp.cl/inicio/wp-content/uploads/2013/01/ Sistema-de-Reparto-Fundaci\%C3\%B3n-Sol.pdf accesado el 17 de junio de 2017.

Garretón, M. (2012). "El gobierno de Michelle Bachelet”. En Neoliberalismo corregido y progresismo limitado: Los gobiernos de la Concertación en Chile, 1990-201,. Santiago de Chile: Ed. ARCIS. accesado el 21 de mayo de 2017. http://biblioteca.clacso.edu.ar/ clacso/coediciones/20121121122525/NeoliberalismoCorregido.pdf 
Madera, N. (2011). La política de inclusión previsional en Argentina: Análisis y perspectivas. Ponencia presentada en el Sexto Congreso Argentino de Administración Pública, Resistencia.

Marquez, I. (2004). Impacto de la reforma previsional de 1981 en los beneficios de los afiliados. Santiago de Chile. Universidad de Chile, Facultad de Ciencias Sociales, Escuela de Posgrado, http://www.tesis.uchile.cl/tesis/uchile/2004/marquez_i/sources/marquez_i.pdf accesado el 15 de marzo de 2017. República de Chile, Resumen Ejecutivo Informe Consejo Reforma Previsional (2006) Informe. Resumen Ejecutivo. Descargado de https://www.bcn.cl/actualidad_legislativa/temas_portada.2006-07-07.2984660535/resumen_ejecutivo_cmarcel1.pdf

República de Chile, Ministerio de Desarrollo Social (2015). Descargado de http://www.ministeriodesarrollosocial.gob.cl/pdf/upload/IDS2.pdf

Moreno, G. (2017). En defensa del modelo. Buenos Aires: Sudamericana.

\section{Entrevistas}

Asesor Previsional de Proyecto Sur, comunicación personal, 2014

Demian Panigo, 2010, entrevista recuperada de http://www.nuevaszonceras.com.ar/ noticias/82-movil-como-esta-ley-vacia-la-anses

Guillermo Moreno, 2015, entrevista recuperada de https://www.youtube.com/ watch? $\mathrm{v}=\mathrm{g} \_2 \mathrm{KtWGSQ}$ c0\&t $=61 \mathrm{~s}$

\section{Fuentes digitales}

Ley 3.500. Diario Oficial de la República de Chile, 13 de noviembre de 1980. https:// www.leychile.cl/Navegar?idNorma=7147_accesado el 22 de junio de 2017. Ley 25.994. Boletín Oficial de la República Argentina, 7 de enero de 2005. Recuperado de http:// servicios.infoleg.gob.ar/infolegInternet/anexos/100000-104999/102726/norma.htm accesado el 24 de junio de 2017.

Ley 24.241. Boletín Oficial de la República Argentina, 18 de octubre de 1993. Recuperado de http://servicios.infoleg.gob.ar/infolegInternet/anexos/0-4999/639/norma. htm accesado el 30 de junio de 2017.

Ley 20.255. Diario Oficial de la República de Chile, 17 de marzo de 2008. Recuperado de https://www.leychile.cl/Navegar?idNorma=269892_accesado el 1 de junio de 2017.

Ley 26.425. Boletín Oficial de la República Argentina, 9 de diciembre de 2008. Recuperado de http://servicios.infoleg.gob.ar/infolegInternet/anexos/145000-149999/148141/ norma.htm accesado el 10 de junio de 2017. 
Ley 26.970. Boletín Oficial de la República Argentina, 10 de septiembre de 2014. Recuperado de: http://servicios.infoleg.gob.ar/infolegInternet/anexos/230000-234999/234847/norma. htm accesado el 12 de junio de 2017.

SVS educa. Portal de educación financiera:

http://www.svs.cl/educa/602/w3-article-1731.html accesado el 11 de junio de 2017.

Superintendencia de Pensiones:

http://www.spensiones.cl/portal/orientacion/580/w3-article-5786.html accesado el 11 de junio de 2017.

\section{Cómo citar este artículo}

Oesterheld, Francisco (2018) "Los proyectos legislativos previsionales en Argentina y Chile durante el período 2005-2010. Un estudio comparado”. Revista Perspectivas de Políticas Públicas Vol 7 No14:347-365 